\title{
CALCULATION OF A VELOCITY DISTRIBUTION FROM PARTICLE TRAJECTORY END-POINTS
}

\author{
By L. A. Rasmussen \\ (U.S. Geological Survey, Tacoma, Washington 98402, U.S.A.)
}

\begin{abstract}
AвStRACt. The longitudinal component of the velocity of a particle at or near a glacier surface is considered. its position as a function of time being termed its trajectory. Functional relationships are derived for obtaining the trajectory from the spatial distribution of velocity and for obtaining the velocity distribution from the trajectory. It is established that the trajectory end-points impose only an integral condition on the velocity distribution. and that no individual point on the velocity distribution can be determined if only the end-points are known. An example is given of a deduced velocity distribution that is consistent with (although not uniquely determined by) the end points of several trajectories on the lower reach of Columbia Glacier, Alaska. It is shown that constructing a velocity distribution by assigning the average trajectory velocity to the trajectory mid-point can be subject to errors of several per cent for velocity distribution features that are typical of actual glaciers. The error in this method is determined, and closed-form expressions for the trajectory are obtained, for linear velocity distributions and for two classes of second-degree distributions. The class of functions is identified to which the velocity distribution must belong for this error to be zero.
\end{abstract}

Résumé. Calcul de la distribution des vitesses à partir de la position des extrêmités d'une trajectoire de particule. La composante longitudinale de la vitesse d'une particule à la surface ou près de la surface d'un glacier est examinée, avec sa position en fonction du temps qui limite sa trajectoire. On en déduit les relations qui permettent d'obtenir la trajectoire á partir de la distribution dans l'espace de la vitesse et la distribution des vitesses à partir de la trajectoire. On établit que la connaissance des extrémités de la trajectoire impose seulement une condition intégrale que doit satisfaire la distribution des vitesses si on ne connait que les extrémités de la trajectoire. On donne un exemple de déduction d'une distribution des vitesses qui est cohérente (bien que non déterminée uniquement par elles) avec les positions des extrémités de plusieurs trajectoires sur la langue du Columbia Glacier en Alaska. On montre que la construction d'une distribution des vitesses en attribuant la vitesse moyenne le long d'une trajectoire au point situe au milieu de cette trajectoire peut entrainer des erreurs de plusieurs pour cent dans des modes de distribution des vitesses qui sont courants sur glaciers réels. On calcule l'erreur induite par une telle méthode et on obtient des expressions très convenables pour la trajectoire. pour des distributions linéaires des vitesses et pour deux classes de distributions du second degrè. On identifie la classe des fonctions auxquelles la distribution des vitesses doit appartenir pour que cette erreur soit nulle.

ZuSAMmENFASSUNG. Berechnung einer Geschwindigkeitsverteilung aus den Endpunkten ton Partikelbahnen. Die Längskomponente der Geschwindigkeit eines Partikels auf oder nahe der Oberfläche eines Gletschers wird betrachtet, wobei dessen Lage als Funktion der Zeit aus seiner Bahn bestimmt wird. Zur Gewinnung der Bahn aus der räumlichen Geschwindigkeitsverteilung und umgekehrt werden funktionale Beziehungen hergeleitet. Es wird nachgewiesen, Dass die Endpunkte der Bahnen nur eine Integrationsbedingung für die Geschwindigkeitsverteilung liefern und dass kein spezieller Wert der Geschwindigkeitsverteilung bestimmt werden kann, wenn nur die Endpunkte bekannt sind. Ein Beispiel für eine abgeleitete Geschwindigkeitsverteilung wird gegeben, die mit den Endpunkten einiger Bahnen im unteren Bereich des Columbia Glacier. Alaska. übereinstimmt, wenn sie auch durch diese nicht eindeutig bestimmt wird. Es zeigt sich, dass die Konstruktion einer Geschwindigkeitsverteilung durch die Zuweisung der mittleren Bahngeschwindigkeit an den Bahnmittelpunkt infolge von Besonderheiten in der Geschwindigkeitsverteilung, die typisch für bestehende Gletscher sind, um einige Prozent verfälscht werden kann. Der Fehler dieses Verfahrens wird bestimmt; für die Bahnen bei linearen Geschwindigkeitsverteilungen und bei zwei Klassen von Verteilungen 2. Grades werden geschlossene Ausdrücke hergeleitet. Die Klasse von Funktionen, zu denen die Geschwindigkeitsverteilung gehören muss, wenn dieser Fehler verschwinden soll, wird festgestellt.

\section{INTRODUCTION}

In glaciological field practice, surface motion is observed using standard surveying 
techniques or photogrammetry to measure the displacement of a survey stake or an identifiable surface feature. The expected errors in determining the initial and final positions are reported and are combined to give the expected error in the average velocity, which is computed by dividing the distance between the two positions by the elapsed time, and which is usually assigned to the point midway between the initial and final positions. A spatial distribution of velocity is constructed by interpreting the motion of several survey stakes or surface features in this way.

This method is susceptible to another source of error. The average velocity does not occur at the mid-point of its initial and final positions unless the actual velocity distribution belongs to a restricted class of mathematical functions. Recognizing this source of error may be helpful in reconciling observations of velocity and mass balance through the continuity equation.

The following is an elementary analysis of the case of steady, one-dimensional flow. Only the horizontal component of the longitudinal velocity is considered, and the transverse component is assumed to be zero. It is not necessary that the glacier be in steady state, that is with unchanging geometry, but only that the horizontal velocity distribution be unchanging over the time interval considered. Alternatively, if the actual velocity distribution is changing, the average velocity may be considered instead; it is defined to be a velocity distribution that, if it existed constantly over the time interval, would produce the observed displacements.

\section{USING PARTICLE TRAJECTORIES TO GET A VELOCITY DISTRIBUTION}

Where $x$ is a horizontal coordinate that is positive in the direction of glacier flow, the velocity

$$
v=\frac{\mathrm{d} x}{\mathrm{~d} t}
$$

of a particle is assumed to depend only on $x$; that is,

$$
v=g(x)
$$

where $g(x)$ is a positive, continuous function and $\partial g(x) / \partial t=0$. If the trajectory

$$
x=f(t)
$$

gives the position of a particle on or near the surface at time $t$, and $f(t)$ is a differentiable, increasing function, then, from Equations (1) and (3),

$$
v=f^{\prime}(t)
$$

where $f^{\prime}(\theta) \equiv \mathrm{d} f(\theta) / \mathrm{d} t$. From Equation (3)

$$
t=f^{-1}(x)
$$

where $f^{-1}(\theta)$ is the inverse of $f(\theta)$; that is, $\left.f^{-1} \mid f(\theta)\right]=f\left[f^{-1}(\theta)\right]=\theta$. Now, from Equations (2), (4), and (5),

$$
v=f^{\prime}\left[f^{-1}(x)\right]=g(x) .
$$

Thus, if $f(t)$ is known, $g(x)$ is given by Equation (6).

Also, from Equations (1) and (2),

$$
\frac{\mathrm{d} x}{\mathrm{~d} t}=g(x)
$$


which is a first-order, ordinary differential equation easily integrated by separating variables

$$
\int_{x_{0}}^{x} \frac{\mathrm{d} x}{g(x)}=\int_{t_{0}}^{t} \mathrm{~d} t
$$

where $x_{0}$ is the position of the particle at time $t_{0}$. If

$$
\int \frac{\mathrm{d} x}{g(x)}=G(x)+\Gamma
$$

in which $\Gamma$ is the constant of integration, then, from Equations (8) and (9),

$$
G(x)=G\left(x_{0}\right)+t-t_{0}
$$

or

$$
x=G^{-1}\left[G\left(x_{0}\right)+t-t_{0}\right]
$$

where $G^{-1}[G(\theta)]=G\left[G^{-1}(\theta)\right]=\theta$. Thus, if $g(x)$ is known, $f(t)$ is given by Equation (10).

As shown by Equation (6), the function $x=f(t)$ must be known if the velocity distribution is to be determined; thus, knowing only the end-points $\left(t_{0}, x_{0}\right)$ and $\left(t_{1}, x_{1}\right)$ of a particle trajectory is insufficient for determining $g(x)$. Moreover, as the trajectory end-points represent an integral of $\mathrm{d} x / \mathrm{d} t=g(x)$, they do not determine any particular point on $g(x)$, but rather they impose only an integral condition on it. Therefore, particular points on $v=g(x)$ cannot be obtained from particle trajectory end-points.

The integral condition imposed on the velocity distribution by the trajectory end-points may be derived by introducing

$$
1 / v=r(x)
$$

and substituting for $v$ in Equation (1) to get

$$
\frac{\mathrm{d} x}{\mathrm{~d} t}=1 / r(x)
$$

which is directly integrated to yield

$$
\int_{x_{0}}^{x} r(x) \mathrm{d} x=\int_{t_{0}}^{t} \mathrm{~d} t .
$$

For the trajectory end-points $\left(t_{0}, x_{0}\right)$ and $\left(t_{1}, x_{1}\right)$,

$$
\int_{x_{0}}^{x_{1}} r(x) \mathrm{d} x=t_{1}-t_{0} .
$$

Thus, in deducing a velocity distribution from a set of particle trajectory end-points, a function $r(x)$ must be devised so that it obeys Equation (14) for each trajectory in the set (which in general may all have $t_{0}$ and $t_{1}$ different from one another, provided the assumption $\partial g(x) / \partial t=0$ holds throughout the entire combined time interval). Neither the time-averaged velocity nor the distance-averaged velocity is pertinent here; it is only the Equation (14) integral of the velocity reciprocal that satisfies the governing differential equation (7). The velocity distribution is not 
uniquely determined by the data because, through Equation (14), they impose conditions only on the integral of its reciprocal.

No doubt an optimizing algorithm could be developed that would construct an $r(x)$ satisfying all the required integrals and also minimizing some measure of smoothness, such as the curvature-squared integral, so that the velocity distribution would be free from spurious features not implied by the data. A simple approach to constructing $g(x)$ is first to fit a smooth curve to the $(\bar{x}, \bar{v})$ points from the several pairs of trajectory end-points used (see Equations (16) and (17)), and then to revise it iteratively until its reciprocal $r(x)$ obeys Equation (14) for each pair of end-points.

After an $r(x)$ has been constructed that is consistent with the end-point data, the velocity distribution is obtained easily from

$$
g(x)=1 / r(x) .
$$

Table I lists the end-points of the trajectories of three survey stakes and twelve surface features observed on the lower reach of Columbia Glacier, Alaska. Only the longitudinal component of position is given. Shown in Figure 1 is a numerically constructed $r(x)$ representing the average velocity distribution over the period considered, for during this period there existed a pronounced seasonal variation (personal communication from M. F. Meier and others). The constructed $r(x)$ is consistent with all fifteen trajectories, each of which is indicated by a horizontal line segment from $x_{0}$ to $x_{1}$ at $r=\Delta t / \Delta x$; each line segment has the integral required by Equation (14). It was constructed iteratively so that it agrees with the data of Table I and so that it is smooth, without any spurious features. The slight asynchronism of the fifteen time intervals is accommodated here by supposing that the same time-average velocity distribution exists throughout the entire combined intervals 25 August 1977 through 3 September 1978. The actual $r(x)$ may have a deeper local minimum of $r \approx 1 \mathrm{a} / \mathrm{km}$ at $x \approx 26.5 \mathrm{~km}$. Shown in Figure 2 is the velocity distribution $v=g(x)=1 / r(x)$.

TABLE I. TRAJECTORY END-POINTS FOR THREE SURVEY STAKES AND TWELVE PHOTOGRAMMETRICALLY OBSERVED SURFACE FEATURES ON THE LOWER REACH OF Columbia Glacier

\begin{tabular}{clccc}
\multicolumn{1}{c}{$t_{0}$} & \multicolumn{1}{c}{$t_{1}$} & $\begin{array}{c}\Delta t \\
\mathrm{a}\end{array}$ & $\begin{array}{c}x_{0} \\
\mathrm{~m}\end{array}$ & $\begin{array}{c}x_{1} \\
\mathrm{~m}\end{array}$ \\
& & 1.019 & 21299 & 22138 \\
25 August 1977 & 1 September 1978 & 1.021 & 26746 & 27524 \\
25 August 1977 & 2 September 1978 & 1.021 & 28976 & 29835 \\
25 August 1977 & 3 September 1978 & 1.024 & 21502 & 22385 \\
29 August 1977 & 26 August 1978 & 0.991 & 22095 & 23081 \\
29 August 1977 & 26 August 1978 & 0.991 & 23125 & 24056 \\
29 August 1977 & 26 August 1978 & 0.991 & 2354 & 24449 \\
29 August 1977 & 26 August 1978 & 0.991 & 23540 & 25510 \\
29 August 1977 & 26 August 1978 & 0.991 & 24589 & 27359 \\
29 August 1977 & 26 August 1978 & 0.991 & 26583 & 27331 \\
29 August 1977 & 26 August 1978 & 0.991 & 27587 & 28331 \\
29 August 1977 & 26 August 1978 & 0.991 & 30290 & 31204 \\
29 August 1977 & 26 August 1978 & 0.991 & 31316 & 32347 \\
29 August 1977 & 26 August 1978 & 0.991 & 31916 & 33006 \\
29 August 1977 & 26 August 1978 & 0.991 & 32413 & 33557 \\
29 August 1977 & 26 August 1978 & 0.991 & 32944 & 34176
\end{tabular}




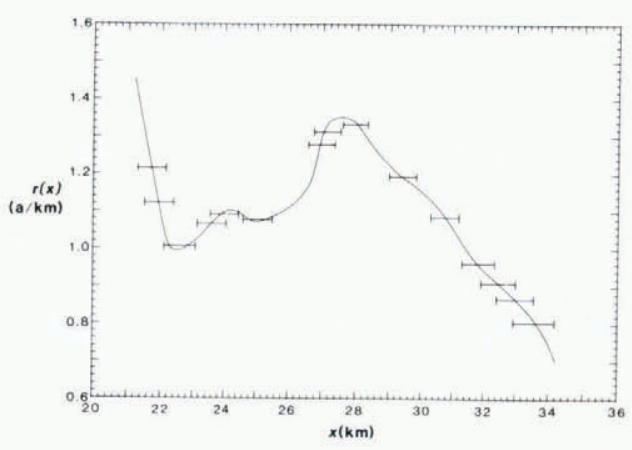

Fig. 1. Reciprocal velocity function $r(x)=1 / v$ constructed numerically to conform with the fifteen trajetories listed in Table I.

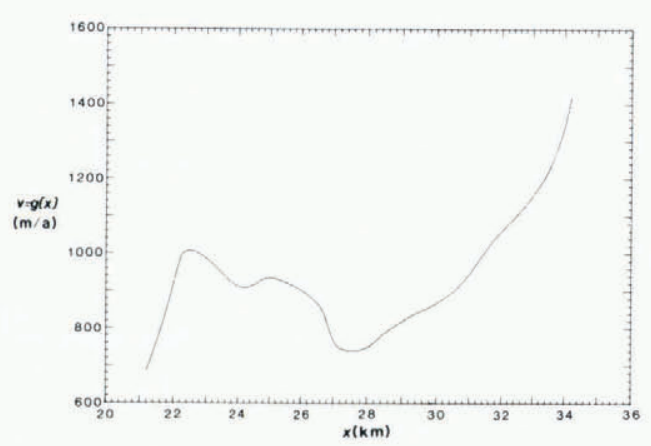

Fig. 2. Velocity distribution $v=g(x)$, which is the reciprocal of the function shown in Figure 1.

\section{THE TRAJECTORY-AVERAGE METHOD}

Although the use of Equation (14) does not uniquely determine the velocity distribution, it does precisely express the entire influence that trajectory end-points have on it. Considered next is the error resulting from using the trajectory-average method to construct it. In this method, the average trajectory velocity

$$
\bar{v}=\frac{x_{1}-x_{0}}{t_{1}-t_{0}} \equiv \frac{\Delta x}{\Delta t}
$$

is assigned to $g(x)$ at the trajectory mid-point

$$
\bar{x}=\frac{x_{0}+x_{1}}{2}
$$

The error in assuming $g(\bar{x})=\bar{v}$ depends on what the actual velocity distribution is. From Equations (8) and (9)

$$
\Delta t=G\left(x_{1}\right)-G\left(x_{0}\right)
$$

and the relative error is

$$
E=\frac{\bar{v}-g(\bar{x})}{g(\bar{x})}=\frac{\Delta x}{g(\bar{x})\left[G\left(x_{1}\right)-G\left(x_{0}\right)\right]}-1 .
$$

This is the error at $\bar{x}$; the error of the trajectory-average method as evaluated by Equation (14) depends on the way a continuous curve might be fitted through $(\bar{x}, \bar{v})$ points calculated by that method from the end-points of several particle trajectories. In investigating the error at $\bar{x}$, the choice of functional forms for $g(x)$ is restricted to those amenable here to manipulation through equations (1) to (10). In each of the three special cases considered, a simple mathematical form for $g(x)$ is assumed, and closed-form expressions are obtained for the trajectory $f(t)$ and the relative error $E$. The complication that sometimes occurs for the coefficients of $g(x)$ and $f(t)$ arises from requiring $f(t)$ to agree with the trajectory end-points.

First considered is the case in which $g(x)$ is a linear function of $x$. It is parametrized by the 
ratio $a$ of the velocities at $x_{0}$ and $x_{1}$; that is, $g\left(x_{1}\right) / g\left(x_{0}\right)=a$. The relevant equations are, for $a \neq 1$,

$$
g(x)=\left(\frac{x-x_{0}}{\Delta t}+\frac{\bar{v}}{a-1}\right) \ln a
$$

and

$$
f(t)=x_{0}+\frac{\Delta x}{a-1}\left[a^{\left(t-t_{0}\right) / \Delta t}-1\right]
$$

and the relative error is

$$
E_{1}(a)=\frac{2}{\ln a}\left(\frac{a-1}{a+1}\right)-1
$$

which is negative for all non-zero gradients $\mathrm{d} v / \mathrm{d} x=(\ln a) / \Delta t$. In the trivial $a=1$ case: $g(x)=\bar{v}, f(t)=x_{0}+\left(t-t_{0}\right) \bar{v}$, and $E_{1}=0$. Because $E_{1}(a)$ is an even function of $\ln a$, it depends only on the magnitude of the gradient, not on its direction. It is shown as Figure 3 ; it approaches -1 as $a$ goes to infinity or zero, and it has an inflection point at $E_{1}(2.169)=-0.0471$. In Figure 4 are shown $f(t)$ for selected $a$.

That $E_{1}(a)$ is independent of the direction of the velocity gradient is a special case of the independence, for any $g(x)$, of the relative error at $\bar{x}$ from the direction in which a particle traverses a feature of $g(x)$; that is, the relative error at $\bar{x}$ is unchanged if $g(x)$ is reflected about $x=\bar{x}$. This is easily established by setting $\hat{g}(x)=g\left(x_{0}+x_{1}-x\right)$, from which $\hat{g}(\bar{x})=g(\bar{x})$ and, from Equation (9)

$$
\begin{aligned}
\hat{G}\left(x_{1}\right)-\hat{G}\left(x_{0}\right) & =\int_{x_{0}}^{x_{1}} \frac{\mathrm{d} x}{\hat{g}(x)}=\int_{x_{0}}^{x_{1}} \frac{\mathrm{d} x}{g\left(x_{0}+x_{1}-x\right)} \\
& =-\int_{x_{1}}^{x_{0}} \frac{\mathrm{d}\left(x_{0}+x_{1}-x\right)}{g\left(x_{0}+x_{1}-x\right)}=\int_{x_{0}}^{x_{1}} \frac{\mathrm{d}\left(x_{0}+x_{1}-x\right)}{g\left(x_{0}+x_{1}-x\right)} \\
& =G\left(x_{1}\right)-G\left(x_{0}\right)
\end{aligned}
$$

so that, from Equation (19), $\hat{E}=E$.

Second is considered the case of a velocity extremum in which $g(x)$ is taken to be a quadratic function of $x$. It is parameterized by the ratio $b$ of the velocity at the mid-point to the velocity at the end-points; that is, $g(\bar{x}) / g\left(x_{0}\right)=g(\bar{x}) / g\left(x_{1}\right)=b$. The velocity distribution is

$$
g(x)=g(\bar{x})\left[1+\left(\frac{1-b}{b}\right)\left(\frac{x-\bar{x}}{\Delta x / 2}\right)^{2}\right] .
$$

Defining

$$
\left.\begin{array}{l}
B_{1}=\sqrt{(1-b) / b}, \\
B_{2}=\sqrt{(b-1) / b}, \\
B_{3}=\left(1+B_{2}\right) /\left(1-B_{2}\right),
\end{array}\right\}
$$




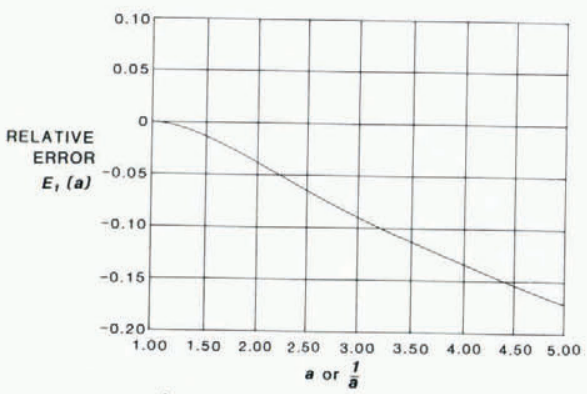

Fig. 3. Relative error $E_{1}(a)$ for linear velocity distribution $v=g(x)$ where $a=g\left(x_{1}\right) / g\left(x_{0}\right)$.

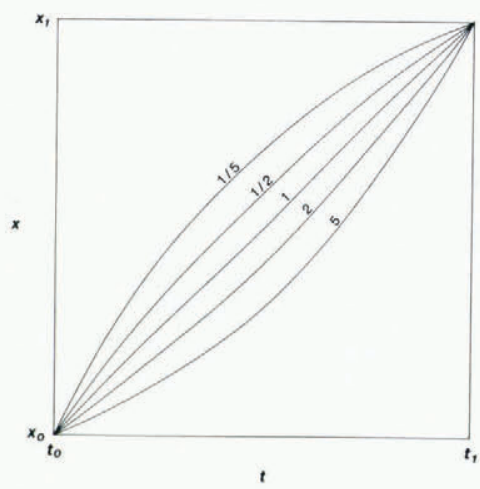

Fig. 4. Particle trajectories $x=f(t)$ from linear velocity distributions $v=g(x)$ for $a=g\left(x_{1}\right) / g\left(x_{0}\right)$ as indicated.

the value of $g(\bar{x})$ that is consistent with the trajectory end-points

$$
g(\bar{x})= \begin{cases}\bar{v}\left(\tan ^{-1} B_{1}\right) / B_{1} & b<1 \\ \bar{v}\left(\ln B_{3}\right) /\left(2 B_{2}\right) & b>1,\end{cases}
$$

the trajectory is

$$
f(t)= \begin{cases}\bar{x}+\frac{\Delta x}{2 B_{1}} \tan \left[\frac{2(t-\bar{t})}{\Delta t} \tan ^{-1} B_{1}\right] & b<1 \\ \bar{x}+\frac{\Delta x}{2 B_{2}}\left[B_{3}^{2(t-\bar{t}) / \Delta t}-1\right] /\left[B_{3}^{2(t-\bar{t}) / \Delta t}+1\right] & b>1,\end{cases}
$$

and the relative error is

$$
E_{2}(b)= \begin{cases}\left(B_{1} / \tan ^{-1} B_{1}\right)-1 & b<1 \\ \left(2 B_{2} / \ln B_{3}\right)-1 & b>1 .\end{cases}
$$

Figure 5 shows $g(x) / g\left(x_{0}\right)$ for selected $b$, and Figure 6 gives the corresponding $f(t)$. Figure 7 gives $E_{2}(b)$ as the solid curve.

Third is considered another form for a velocity extremum in which $g(x)$ is taken to be a segment of a conic section. This functional form is included because the form of Equation (24) does not span all second-degree possibilities. It is parameterized by the ratio $c$ of the velocity at the mid-point to the velocity at the end-points; that is, $g(\bar{x}) / g\left(x_{0}\right)=g(\bar{x}) / g\left(x_{1}\right)=c$. The velocity distribution is

$$
g(x)=g(\bar{x}) \sqrt{1+\left(\frac{1-c^{2}}{c^{2}}\right)\left(\frac{x-\bar{x}}{\Delta x / 2}\right)^{2}} .
$$

Defining

$$
\begin{aligned}
C_{1} & =\sqrt{c^{2}-1} / c, \\
C_{2} & =\sqrt{1-c^{2}} / c, \\
C_{3} & =\left(1+c C_{2}\right) /\left(1-c C_{2}\right),
\end{aligned}
$$




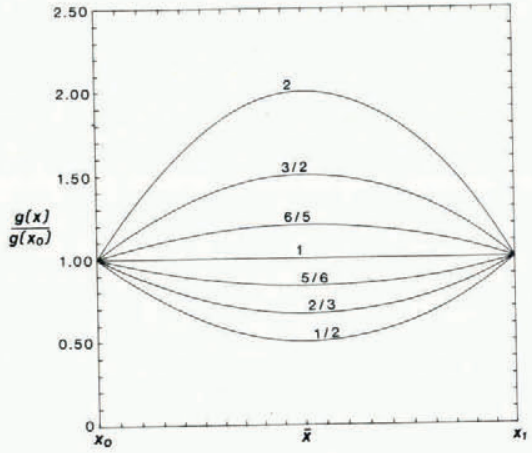

Fig. 5. Quadratic velocity distributions $v=g(x)$ for $b=g(\bar{x}) / g\left(x_{0}\right)$ as indicated.

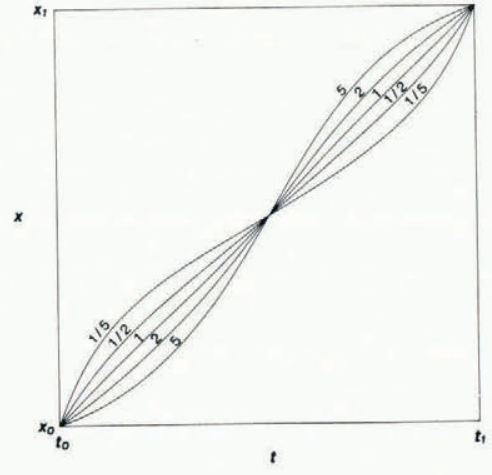

Fig. 6. Particle trajectories $x=f(t)$ from quadratic velocity distributions $v=g(x)$, for $b=g(\bar{x}) / g\left(x_{0}\right)$ as indicated.

the value of $g(\bar{x})$ that is consistent with the trajectory end-points is

$$
g(\bar{x})= \begin{cases}\bar{v}\left(\sin ^{-1} C_{1}\right) / C_{1} & c>1 \\ \bar{v}\left(\ln C_{3}\right) /\left(2 C_{2}\right) & c<1,\end{cases}
$$

the trajectory is

$$
f(t)= \begin{cases}\bar{x}+\frac{\Delta x}{2 C_{1}} \sin \left[\frac{2(t-\bar{t})}{\Delta t} \sin ^{-1} C_{1}\right] & c>1 \\ \bar{x}+\frac{\Delta x}{4 C_{2}}\left[C_{3}^{(t-\bar{t}) / \Delta t}-C_{3}^{-(t-\bar{t}) / \Delta t}\right] & c<1,\end{cases}
$$

and the relative error is

$$
E_{3}(c)= \begin{cases}\left(C_{1} / \sin ^{-1} C_{1}\right)-1 & c>1 \\ \left(2 C_{2} / \ln C_{3}\right)-1 & c<1 .\end{cases}
$$

When $c>1$ the conic is an ellipse, and when $c<1$ it is a hyperbola. Figure 8 shows $g(x) / g\left(x_{0}\right)$ for selected $c$, and Figure 9 gives the corresponding $f(t)$. Figure 7 gives $E_{3}(c)$ as the dashed curve.

Although it is difficult to determine the error in assigning $(\bar{x}, \bar{v})$ to $g(x)$ for arbitrary $g(x)$, it is easy to determine the class of functions to which $g(x)$ must belong if $g(\bar{x})=\bar{v}$. Equation (14) may be written

$$
\int_{-\Delta x / 2}^{\Delta x / 2} \hat{r}(x-\bar{x}) \mathrm{d}(x-\bar{x})=\Delta t
$$

where $\hat{r}(x-\bar{x})=r(x)$. Since any function can be expressed as the sum of an odd function and an even function, let

$$
\hat{r}(x-\bar{x})=\hat{r}_{1}(x-\bar{x})+\hat{r}_{2}(x-\bar{x})
$$

where $\hat{r}_{1}(\bar{x}-x)=-\hat{r}_{1}(x-\bar{x})$, from which $\hat{r}_{1}(0)=0$, and $\hat{r}_{2}(\bar{x}-x)=\hat{r}_{2}(x-\bar{x})$. If $g(\bar{x})=\bar{v}$, then 


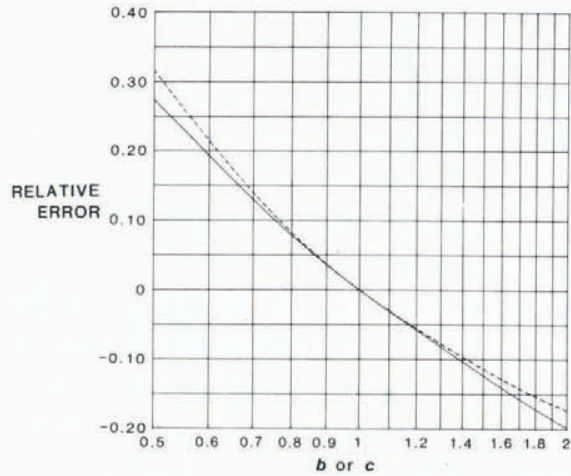

Fig. 7. Relative error, $E_{2}(b)$ for quadratic velocity distribution (solid curve) and $E_{3}(c)$ for conic velocity distribution (dashed curve), where $v=g(x)$ and $b, c=g(\bar{x}) / g\left(x_{0}\right)$.

$r(\hat{i})=\hat{r}(0)=\hat{r}_{2}(0)=1 / \bar{v}$, and Equation (34) may be written

$\int_{-\Delta x / 2}^{\Delta \Delta x / 2} \hat{r}_{1}(x-\bar{x}) \mathrm{d}(x-\bar{x})+\int_{-\Delta x / 2}^{-\Delta x / 2}\left[\hat{r}_{2}(x-\bar{x})-1 / \bar{v}\right] \mathrm{d}(x-\bar{x})+\int_{-\Delta x / 2}^{-\Delta x / 2}(1 / \bar{v}) \mathrm{d}(x-\bar{x})=\Delta t$.

Because the first integral vanishes identically and the third integral is $\Delta x / \bar{v}=\Delta t$, the second integral must also vanish; this gives the other condition on the velocity distribution for it to include $(\bar{x}, \bar{v})$. The simplest form for such $g(x)$ then is $\hat{r}_{1}(x-\bar{x})=0$ and $\hat{r}_{2}(x-\bar{x})=1 / \bar{v}$, which gives $g(\bar{x})=\bar{v}$. This is the trivial case with zero gradient of the linear, quadratic, and conic functions examined above for $g(x)$. Increasing the degree of $r(x)$ by one gives

$$
g(x)=1 /[s(x-\bar{x})+1 / v]
$$

where $s$ is a constant, which is a hyperbola with gradient $\mathrm{d} v / \mathrm{d} x=-s v^{2}$.

Equation (37) can be interpreted glaciologically if it is assumed that the glacier flux $Q$ obeys

$$
Q=F g(x) h(x)
$$

where $h(x)$ is the glacier thickness and $F$ is a constant, and if it is assumed that over some $x$ interval $Q$ is also constant and $h(x)$ is linear, then over that interval $g(x)$ has the same form as in

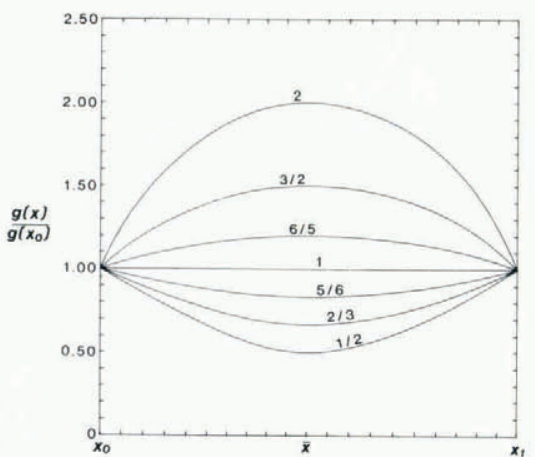

Fig. 8. Conic velocity distributions $v=g(x)$ for $c=g(\bar{x}) / g\left(x_{0}\right)$ as indicated.

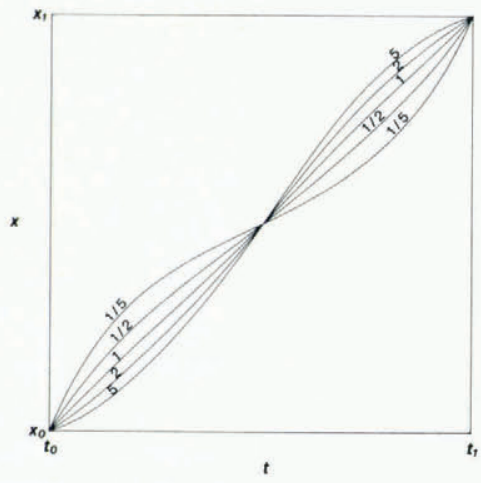

Fig. 9. Particle trajectories $x=f(t)$ from conic velocity distributions $v=g(x)$, for $c=g(\bar{x}) / g\left(x_{0}\right)$ as indicated. 
Equation (37) and $g(\bar{x})=\bar{v}$. In fact, the conditions on $Q$ and $F$ can be relaxed slightly; $Q$ and $F$ can both vary with $x$ as long as the ratio $Q(x) / F(x)$ is constant. This special case, however, should not be embraced as a basis for neglecting the error to which the trajectory-average method is susceptible; in spite of this effect, the curves of Figures 3 and 7 still exhibit errors of several per cent, and so does the actual glacier velocity distribution discussed next. It is mentioned only to put Equation (37) into a glaciological context, not to suggest that the conditions occur in actual glaciers with any particular frequency.

An actual glacier velocity distribution provides another example of the error in the trajectory-average method. The velocity distribution for the ice-fall reach of Columbia Glacier (from unpublished data taken in 1982 by M. F. Meier and others) is shown as the solid curve in Figure 10; it is much more complex than the one for the lower reach shown in Figure 2. If the velocity distribution is assumed to be unchanging from time $t_{0}$ until time $t_{1}=t_{0}+\Delta t$, then for a survey stake initially at some position $x_{0}$, its final position $x_{1}$ is given by Equation (14); the average trajectory velocity, given by equation (16), is assigned to the trajectory mid-point, given by Equation (17). If a dense array of hypothetical survey stakes were assumed to exist at time $t_{0}$, and if the solid curve in Figure 10 is assumed to be the actual velocity distribution, then the velocity distribution that would be produced by the trajectory-average method can be developed; the dashed curve in Figure 10 is the distribution so developed for one year $\Delta t$. The relative error $E$, which is given by Equation (19), can also be computed at the mid-point of the trajectory of each hypothetical stake; the $E(x)$ for the dashed curve in Figure 10 is shown as the dashed curve in Figure 11, which also gives the $E(x)$ for $\Delta t=2.0,0.5$, and 0.25 a.

Several conclusions can be drawn from this actual case. First, the velocity distribution arising from the trajectory-average method is one that yields a subdued curve, especially in its understatement of the extrema; however, the extreme values of the associated error distribution are displaced from those extrema (compare the dashed curves in Figures 10 and 11). Second, at any $x$, the error is roughly proportional to $\Delta t$. Third, the error cannot be avoided or reduced by some stake placement technique, because this case has been analyzed by assuming a continuum of hypothetical stakes, although a very sparse stake spacing could fail entirely to detect features in the velocity distribution. Therefore, if a field program is to be designed to reduce the error in

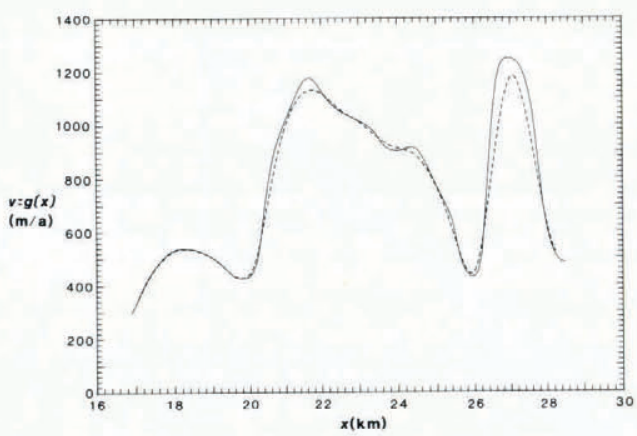

Fig. 10. Velocity distribution for the ice-fall reach of the Columbia Glacier, both the actual (solid curve) and that which would be produced by the trajectoryaverage method (dashed curve).

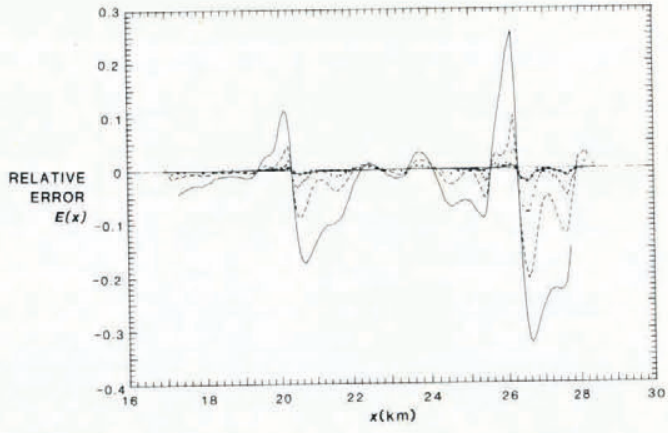

Fig. 11. Relative error $E(x)$ in applying the trajectoryaverage method to the velocity distribution shown as the solid curve in Figure 10 for $\Delta t=2.0 a$ (solid curve), $1.0 a$ (dashed curve), 0.5a (dotted curve), and $0.25 a$ (crossed curve). 
using the trajectory-average method, only shortening the period between position measurements will have any effect, not the stake placement pattern. The error depends only on the actual velocity distribution a stake traverses in a particular time $\Delta t$, not on the position of some other stake. Easier than shortening the measurement period would be using Equation (14) in the construction of the velocity distribution, rather than using the trajectory-average method.

\section{SUMMARY}

The trajectory end-points customarily observed in glacier field programs impose only an integral condition on the velocity distribution. Therefore, they do not uniquely determine it, or any individual points on it.

The trajectory-average method, in which the average trajectory velocity $\bar{v}$ is assigned to the trajectory mid-point $\bar{x}$, has an inherent error. For a one-year interval $\Delta t$ between position measurements, this error can reach several per cent for velocity distribution features that are typical of actual glaciers. The case of the ice-fall reach of Columbia Glacier suggests that this error is roughly proportional to $\Delta t$.

The only field program tactic that can be used to reduce this error is to shorten $\Delta t$. Although spatially enriching a stake array will give more information about the velocity distribution, it cannot reduce this error.

The $(\bar{x}, \bar{v})$ points are useful in making a first approximation to the velocity distribution. The reciprocal of a smooth curve through them should be revised as necessary to make it obey Equation (14) for all trajectory end-points used. The reciprocal of this revised reciprocal curve is then the velocity distribution.

MS. received I September 1982 and in revised form 29 November 1982

\section{APPENDIX}

THE development of Equations (20)-(22), (26)-(28), and (31)-(33) requires considerable algebraic manipulation. Because detailing all the steps would be voluminous, only the principal intermediate quantities are given here.

(A) Beginning with a general linear equation in $x$, and requiring that both the $a$-ratio and Equation (14) be satisfied, the coefficients are determined to be those of Equation (20). Defining $A_{1}=\Delta x /(a-1)$ and $A_{2}=\Delta t / \ln a$. they

so that, from Equation (9),

$$
g(x)=\left(x-x_{0}+A_{1}\right) / A_{2},
$$

from which

$$
G(x)=A_{2} \ln \left(x-x_{0}+A_{1}\right),
$$

and

$$
G\left(x_{0}\right)=A_{2} \ln A_{1}
$$

$$
G^{-1}(\theta)=x_{0}-A_{1}+a^{\theta / \Delta t} .
$$

From Equation (10), along with equations $(\mathrm{A}-3)$ and $(\mathrm{A}-4)$,

$$
f(t)=x_{0}+A_{1}\left[a^{\left(t-t_{0}\right) / \Delta t}-1\right]
$$


which is equivalent to Equation (21), and from which

$$
f^{\prime}(t)=\frac{A_{1}}{A_{2}} a^{\left(t-t_{0}\right) / \Delta t}
$$

and

$$
f^{-1}(x)=t_{0}+A_{2} \ln \left[1+\left(x-x_{0}\right) / A_{1}\right] .
$$

(B) Equation (24) is a quadratic in $x$ that already satisfies the $b$-ratio. When $b<1$,

$$
g(\bar{x})=\bar{v}\left(\tan ^{-1} B_{1}\right) / B_{1}
$$

supplies the coefficient in Equation (24) so that it satisfies Equation (14). From Equation (9)

$$
G(x)=\frac{\Delta t}{2} \tan ^{-1}\left[\frac{2 B_{1}(x-\bar{x})}{\Delta x}\right] / \tan ^{-1} B_{1},
$$

from which $G\left(x_{0}\right)=-\Delta x / 2$ and

$$
G^{-1}(\theta)=\bar{x}+\frac{\Delta x}{2 B_{1}} \tan \left(\frac{2 \theta \tan ^{-1} B_{1}}{\Delta t}\right) .
$$

From Equation (10), $f(t)=G^{-1}(t-7)$, which is equivalent to Equation (27) for $b<1$, and from which $f^{-1}(x)=\bar{t}+G(x)$ and

$$
f^{\prime}(t)=\bar{v} \frac{\tan B_{1}}{B_{1}} / \cos ^{2}\left[\frac{2(t-\tau) \tan ^{-1} B_{1}}{\Delta t}\right] .
$$

When $b>1$, the value of the inverse tangent for complex argument gives $B_{1} / \tan ^{-1} B_{1}=2 B_{2} / \ln B_{3}$. Then

$$
\begin{gathered}
g(\bar{x})=\bar{v}\left(\ln B_{3}\right) /\left(2 B_{2}\right), \\
G(x)=\frac{\Delta t}{2} \ln \left[\frac{\Delta x+2(x-\bar{x}) B_{2}}{\Delta x-2(x-\bar{x}) B_{2}}\right] / \ln B_{3}, \\
G^{-1}(\theta)=\bar{x}+\frac{\Delta x}{2 B_{2}}\left(B_{3}^{2 \theta / \Delta t}-1\right) /\left(B_{3}^{2 \theta / \Delta t}+1\right), \\
f^{\prime}(t)=\frac{2 \bar{v} \ln B_{3}}{B_{2}} B_{3}^{2(t-t) / \Delta t} /\left[B_{3}^{2(t-t) / \Delta t}+1\right] .
\end{gathered}
$$

(C) Equation (29) is a conic in $v$ and $x$ that already satisfies the $c$-ratio. When $c>1$,

$$
g(\bar{x})=\bar{v}\left(\sin ^{-1} C_{1}\right) / C_{1}
$$

supplies the coefficient in Equation (29) so that it satisfies Equation (14). From Equation (9)

$$
G(x)=\frac{\Delta t}{2} \sin ^{-1}\left[\frac{2(x-\bar{x}) C_{1}}{\Delta x}\right] / \sin ^{-1} C_{1},
$$

from which $G\left(x_{0}\right)=-\Delta t / 2$ and

$$
G^{-1}(\theta)=\bar{x}+\frac{\Delta x}{2 C_{1}} \sin \left(\frac{2 \theta \sin ^{-1} C_{1}}{\Delta t}\right) .
$$

From Equation (10), $f(t)=G^{-1}(t-7)$, which is equivalent to Equation (32) for $c>1$, and from which $f^{-1}(x)=t+G(x)$ and

$$
f^{\prime}(t)=\frac{\bar{v} \sin ^{-1} C_{1}}{C_{1}} \cos \left[\frac{2(t-\bar{t}) \sin ^{-1} C_{1}}{\Delta t}\right] .
$$


When $c<1$, the value of the inverse sine for complex argument gives $C_{1} / \sin ^{-1} C_{1}=2 C_{2} / \ln C_{3}$. Then

$$
\begin{gathered}
g(\bar{x})=\bar{v}\left(\ln C_{3}\right) /\left(2 C_{2}\right), \\
G(x)=\frac{\Delta t}{\ln C_{3}} \ln \left\{\frac{2(x-\bar{x}) C_{2}}{\Delta x}+\sqrt{1+\left[\frac{2(x-\bar{x}) C_{2}}{\Delta x}\right]^{2}}\right\}, \\
G^{-1}(\theta)=\bar{x}+\frac{\Delta x}{4 C_{2}}\left(C_{3}^{\theta / \Delta t}-C_{3}^{-\theta / \Delta t}\right), \\
f^{\prime}(t)=\frac{\bar{v} \ln C_{3}}{4 C_{2}}\left[C_{3}^{(t-\bar{t}) / \Delta t}+C_{3}^{-(t-t) / \Delta t}\right] .
\end{gathered}
$$

